ARTICLE

https://doi.org/10.1038/s41467-019-11961-9

\title{
Tracking the ultrafast motion of an antiferromagnetic order parameter
}

\author{
Christian Tzschaschel (1) ${ }^{1}$, Takuya Satoh (i) ${ }^{2,3} \&$ Manfred Fiebig ${ }^{1}$
}

The unique functionalities of antiferromagnets offer promising routes to advance information technology. Their compensated magnetic order leads to spin resonances in the THz-regime, which suggest the possibility to coherently control antiferromagnetic (AFM) devices orders of magnitude faster than traditional electronics. However, the required time resolution, complex sublattice interactions and the relative inaccessibility of the AFM order parameter pose serious challenges to studying AFM spin dynamics. Here, we reveal the temporal evolution of an AFM order parameter directly in the time domain. We modulate the AFM order in hexagonal $\mathrm{YMnO}_{3}$ by coherent magnon excitation and track the ensuing motion of the AFM order parameter using time-resolved optical second-harmonic generation. The dynamic symmetry reduction by the moving order parameter allows us to separate electron dynamics from spin dynamics. As transient symmetry reductions are common to coherent excitations, we have a general tool for tracking the ultrafast motion of an AFM order parameter.

\footnotetext{
${ }^{1}$ Department of Materials, ETH Zurich, 8093 Zurich, Switzerland. ${ }^{2}$ Department of Physics, Tokyo Institute of Technology, Tokyo 152-8551, Japan.

${ }^{3}$ Department of Physics, Kyushu University, Fukuoka 819-0395, Japan. Correspondence and requests for materials should be addressed to

C.T. (email: christian.tzschaschel@mat.ethz.ch)
} 
$\mathrm{R}$ ecently, the appealing properties of antiferromagnets culminated in the demonstration of both optically ${ }^{1}$ and electrically ${ }^{2}$ induced switching of the AFM order. This illustrates the general usefulness of antiferromagnets for future applications ${ }^{3,4}$, yet the dynamics occurring during the switching process remain uncertain as time-resolved (TR) access to the AFM order parameter is notoriously difficult. Consequently, detecting its motion in the time domain often relies on measuring one spin component, such as a transiently occuring uncompensated magnetic moment, and extrapolating the full orderparameter dynamics from models ${ }^{1}$. A detailed study of all spin components during a ferromagnetic spin precession, however, revealed unexpected insights ${ }^{5,6}$. Thus, a dependable analysis of the non-trivial spin dynamics during switching events in antiferromagnets necessitates direct and TR experimental access to all the order-parameter components.

While the uncompensated component is optically accessible by the Faraday effect, detection of the compensated part of the order parameter usually depends on more indirect processes, like magnetic linear birefringence, which is quadratic in the sublattice magnetisation. This leads to a loss of directionality and, thereby, of the ability to distinguish between spin-reversed domain states $^{2,7-9}$. Here, optical second-harmonic generation (SHG), i.e., frequency doubling of a light wave in a material, is a powerful, symmetry-sensitive technique that can couple linearly to the AFM order parameter, thus maintaining its directional information ${ }^{10,11}$. The leading SHG contribution is given by $P_{i}(2 \omega)=\epsilon_{0} \mathcal{X}_{i j k} E_{j}(\omega) E_{k}(\omega)$, where $\epsilon_{0}$ is the vacuum permittivity, $E$ is the electric field of the incoming light wave, $\mathcal{X}$ is the secondorder susceptibility tensor and $P$ is the induced polarisation oscillating at twice the frequency $\omega$ of the incident light ${ }^{12}$. Owing to the direct coupling of $\mathcal{X}$ to the AFM order parameter, SHG is ideal for the static characterisation of antiferromagnets ${ }^{11,13,14}$. Despite this and despite the instantaneous nature of the SHG process, which allows probing ultrafast processes, TR SHG studies of optically induced dynamics are scarce. The methodical difficulty to discriminate between genuine magnetic-order-parameter dynamics, affecting $\mathcal{X}_{i j k}$, and electron dynamics, affecting the linear optical properties at $\omega$ and $2 \omega$, hampers the interpretation of TR SHG measurements ${ }^{15}$. In fact, distinguishing between order-parameter and electron dynamics is commonly believed to be a "practical impossibility" 16 . This is particularly true for thermal excitations, where redistribution of resonantly excited charge carriers leads to substantial changes in the linear optical properties $^{17-20}$.

An optical excitation, however, can also act as a non-thermal stimulus to the magnetic order. A well-known example is the inverse Faraday effect (IFE) ${ }^{16}$, where a circularly polarised laser pulse generates a longitudinal effective magnetic field $H_{\mathrm{IFE}}$. This field exists for the laser pulse duration only. The impulsive, nonresonant excitation results in a well-defined initial phase of the spin precession. This process is also effective in fully compensated antiferromagnets ${ }^{8,21-26}$. Such a simple fundamental excitation is an ideal test case for us. Its well-understood dynamics allow us to demonstrate how to separate thermal electron dynamics from non-thermal spin dynamics and how to obtain the full orderparameter trajectory.

Here, we use a combination of Faraday rotation and SHG to track the order-parameter motion during a coherent spin precession in antiferromagnetic $\mathrm{YMnO}_{3}$. We find that the coherent spin precession transiently reduces the point-group symmetry, which leads to the emergence of new SHG tensor components. We conclude this from a periodic modulation of the SHG anisotropy. In contrast to the Faraday rotation, which is sensitive to the out-of-plane spin component, we show that the SHG modulation reflects the in-plane spin component. Moreover, we quantify both the in-plane and out-of-plane spin-canting angles, where we find a pronounced ellipticity of the spin precession. With this proof-of-concept demonstration, we show that TR SHG is a valuable tool for the study of AFM spin dynamics providing complementary information that is inaccessible by other techniques.

\section{Results}

Experimental details. We choose hexagonal $\mathrm{YMnO}_{3}$ as a model system. The material is ferroelectric below $T_{\mathrm{C}} \approx 1250 \mathrm{~K}^{27}$. The three $\mathrm{Mn}^{3+}$ sublattices order antiferromagnetically below the Néel temperature $T_{\mathrm{N}} \approx 70 \mathrm{~K}$ and form a quasi-two dimensional triangular lattice with a fully compensated spin structure. The spins in the sublattices point along the local $x$ axes ${ }^{11}$. The magnetic space group of the ground state is $P 6_{3}^{\prime} \mathrm{cm}^{\prime}$, which gives rise to a SHG contribution coupling bilinearly to the ferroelectric and the AFM order parameter ${ }^{10}$ (Supplementary Fig. 1). The magnetic structure yields three orthogonal magnon modes, named $X$-, $Y$ - and $Z$-mode, which differ in the direction of the transiently appearing oscillating uncompensated magnetisation component. The individual modes can be excited optically and probed selectively depending on the setting of pump and probe-light polarisations ${ }^{23}$. Here, we exemplarily focus on the $Z$-mode, which has $A_{2}$ symmetry ${ }^{28}$.

The optical properties of $\mathrm{YMnO}_{3}$ are well-studied ${ }^{29}$. Owing to the hexagonal crystal structure, the material is optically uniaxial, which enables birefringence-free measurements along the $\mathrm{z}$ axis. The dielectric spectrum is characterised by a charge-transfer transition around $1.6 \mathrm{eV}^{30}$ and low-optical absorption in the near-infrared regime ${ }^{31}$. In order to minimise parasitic, thermally induced, incoherent dynamics ${ }^{24}$, we optically pump the material at $0.97 \mathrm{eV}$, where the residual absorption is $<10 \%$ for our $20 \mu \mathrm{m}$ thin sample and non-thermal excitation via the IFE dominates. We probe the optically induced dynamics at $\hbar \omega=1.22 \mathrm{eV}$, where on the one hand, the high transmittivity enables measurements of the Faraday rotation and on the other hand, efficient SHG is ensured by resonant coupling to a $d-d$ transition of the $\mathrm{Mn}^{3+}$ ions at $2 \hbar \omega^{32}$.

Our setup is sketched in Fig. 1. Via the IFE, a circularly polarised pump pulse cants the spins in the $x y$ plane by an angle $\gamma \mu_{0} H_{\mathrm{IFE}} \tau$, where $\gamma$ is the gyromagnetic ratio, $\mu_{0}$ is the vacuum permeability and $\tau$ is the duration of the effective magnetic field pulse $^{33}$. The transmitted linearly polarised probe pulse is split behind the sample by a dichroic mirror for separate, yet simultaneous, TR detection of the Faraday rotation and of the relative change of the SHG intensity $\left(\eta=\Delta I_{\mathrm{SHG}} / I_{\mathrm{SHG}}\right)$.

Z-mode observation by Faraday rotation and SHG. A typical measurement at $10 \mathrm{~K}$ is shown in Fig. 2. The Faraday rotation in Fig. 2a exhibits sine-like behaviour with a frequency of $\Omega / 2 \pi=95.2 \pm 0.3 \mathrm{GHz}$. The initial phase changes by $\pi$ upon changing the pump helicity, as is expected for a $Z$-mode excitation via the $\mathrm{IFE}^{23}$. In parallel to the Faraday rotation, we measured the SHG response $\eta$ for a probe polarisation enclosing an angle of $\phi \approx 15^{\circ}$ with respect to a crystallographic $\mathrm{x}$ axis. We find a coherent modulation of $\eta$ on an incoherent background. By taking $\left(\eta_{-}-\eta_{+}\right) / 2$ and $\left(\eta_{-}+\eta_{+}\right) / 2$, with the sign indicating the pump-pulse helicity, we can separate helicity-dependent and helicity-independent contributions, respectively (Fig. 2b). We find that the oscillatory component of $\eta$ is fully contained in the helicity-dependent contribution, while the helicity-independent contribution follows a single exponential decay with a timeconstant that is typical for oxide systems ${ }^{17}$. We thus attribute the helicity-independent dynamics to thermalisation processes, in particular also because the quasi-instantaneous effect of possible 


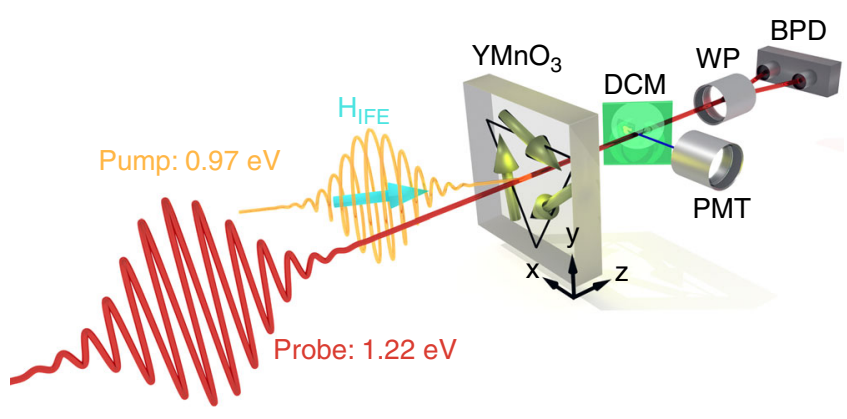

Fig. 1 Illustration of the two-colour pump-probe setup. A circularly polarised $130 \mathrm{fs}$ laser pulse at $0.97 \mathrm{eV}$ induces a spin canting in $\mathrm{YMnO}_{3}$ via the IFE. The subsequent spin precession is probed with a linearly polarised $1.22 \mathrm{eV}$ laser pulse via a simultaneous measurement of the Faraday rotation (using a Wollaston prism (WP) and a balanced photodiode (BPD)) and the SHG signal (using a Glan-Taylor prism and a photomultiplier tube (PMT), to which the frequency doubled light was branched off by a dichroic mirror (DCM))

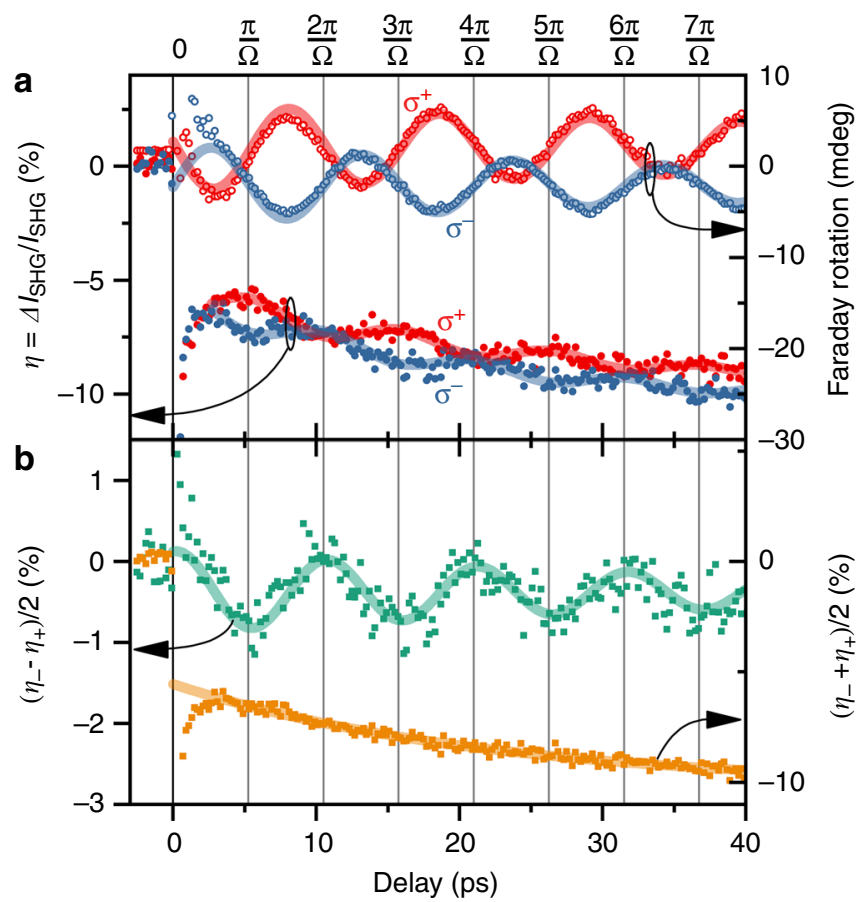

Fig. 2 Experimental observation of coherent spin dynamics by Faraday rotation and SHG. Typical measurement of optically induced spin dynamics by $\sigma^{+}$-polarised (red) and $\sigma^{-}$-polarised (blue) pump pulses. a Open circles represent Faraday rotation. Closed circles show the relative change of SHG intensity. Solid lines are damped sine (Faraday) and cosine (SHG) fits. b Difference (green) and average (orange) of TR SHG changes corroborating helicity-dependent and helicity-independent contributions, respectively. Solid lines represent fits according to a damped cosine (green) and a single exponential decay (orange)

non-thermal, helicity-independent excitations ${ }^{34}$ cannot explain the exponentially progressing, delayed reduction of SHG intensity in Fig. 2b.

The helicity-dependent SHG difference in Fig. 2b reveals a modulation at the same frequency $\Omega$ as the Faraday rotation. We can, therefore, safely conclude that, just like the Faraday rotation, the SHG modulation is based on the optically excited spin precession. Note, however, that the Faraday rotation, which is proportional to the net magnetisation along the $\mathrm{z}$ axis, and the
SHG modulation are out of phase by $\pi / 2$. Hence, the SHG modulation cannot be based on the out-of-plane spin component. Since the linear transmittance of the sample both at $\omega$ and $2 \omega$ does not show any periodic changes either (see Supplementary Fig. 2), we are left with the only option that the SHG modulation reflects the dynamics of the compensated in-plane spin component. We conclude that we observe a modulation of $\mathcal{X}_{i j k}$ induced by dynamic changes of the AFM order parameter $\ell$. There are two fundamentally different mechanisms that can affect $\mathcal{X}_{i j k}$ through $\ell$ : (i) symmetry-conserving dynamics involving amplitude modulations of the existing components $\mathcal{X}_{i j k}$. Examples are longitudinal coherent dynamics ${ }^{35,36}$, changes of the exchange interaction ${ }^{37,38}$ as well as thermally induced quenching dynamics ${ }^{17,20}$. Or (ii) symmetry-changing dynamics leading to the appearance of new components $\mathcal{X}_{i j k}$. Besides, e.g., spinreorientation $^{39}$ and AFM switching processes ${ }^{1}$, this also includes transversal spin dynamics, where precessional spin motion causes a redistribution among the $\mathcal{X}$ components with periodically arising and vanishing contributions.

TR modulation of the SHG anisotropy. We distinguish the two cases by measuring the angle-dependent anisotropy of the SHG signal before and after the excitation. In contrast to Fig. 2, which shows the dynamics of one arbitrary angular component of the SHG anisotropy, measuring the full angle-dependent SHG anisotropy allows us to determine any symmetry changes that might occur. Figure 3a shows the SHG anisotropy prior to excitation. It is in agreement with the $P 6_{3}^{\prime} \mathrm{cm}^{\prime}$ symmetry of the magnetic ground state with the $\mathrm{Mn}^{3+}$ spins oriented along equivalent crystallographic $\mathrm{x}$ axis. The anisotropy exhibits six lobes of amplitude $A$ spaced $60^{\circ}$ each. We define their orientation as $\theta=0^{\circ}$.

The time-dependent SHG intensities at four representative angular components are visualised in Fig. 3b (see "Methods" for details). We find cosine-like modulations with opposite phases at the two sides of a lobe (violet and yellow), but no modulation of the SHG intensity at the minima (blue) or maxima (red). This behaviour cannot be explained by a symmetry-conserving breathing of the SHG anisotropy. Such breathing would manifest itself as an in-phase modulation at the two sides of a lobe and at the maximum. Instead, the observed dynamics are in accordance with a coherent, periodic, small-angle rotation of the SHG anisotropy. By fitting the full SHG anisotropy, we can extract the pump induced changes of the amplitude $A$ and the orientation $\theta$. The results are shown in Fig. $3 c$, d, respectively.

The amplitude $A$ exhibits an exponential decrease of the order of $10 \%$. The change of $A$ cannot be caused by changes of the optical properties as the slight changes of the transmittivity at $\omega$ and $2 \omega$ (see Supplementary Fig. 2) are not sufficient to explain the $10 \%$ decrease of $A$. Instead, the isotropic, i.e., polarisation-independent, behaviour is indicative of an incoherent relaxation. We, therefore, attribute this decay to longitudinal order-parameter dynamics, i.e. to a reduction of the total sublattice magnetisation $g \mu_{B}|S|$ related to the relaxation of photoexcited charge carriers.

As already indicated by Fig. $3 \mathrm{~b}$, $\mathrm{d}$ reveals a striking periodic rotation of the SHG anisotropy. The modulation frequency of $\Delta \theta$ matches that of the Faraday rotation, which confirms the magnetic origin. As illustrated in Fig. 3e, a rotated anisotropy can be decomposed into a linear superposition of two sixfold anisotropies at $\theta=0^{\circ}$ (spins along $\mathrm{x}$ axis, space group $\mathrm{PG}_{3}^{\prime} \mathrm{cm}^{\prime}$, red curve) and $\theta=90^{\circ}$ (spins along y axis, space group $P 6_{3}^{\prime} c^{\prime} m$, blue curve). The superposition corresponds to a coherent deviation of the spins from the $\mathrm{x}$ axis towards the $\mathrm{y}$ axis and, therefore, to a symmetry reduction to $P 6_{3}^{\prime}$ or lower (see Supplementary Fig. 3). With $\tan \alpha=S_{y} / S_{x}$, we can write the dynamic SHG tensor as 

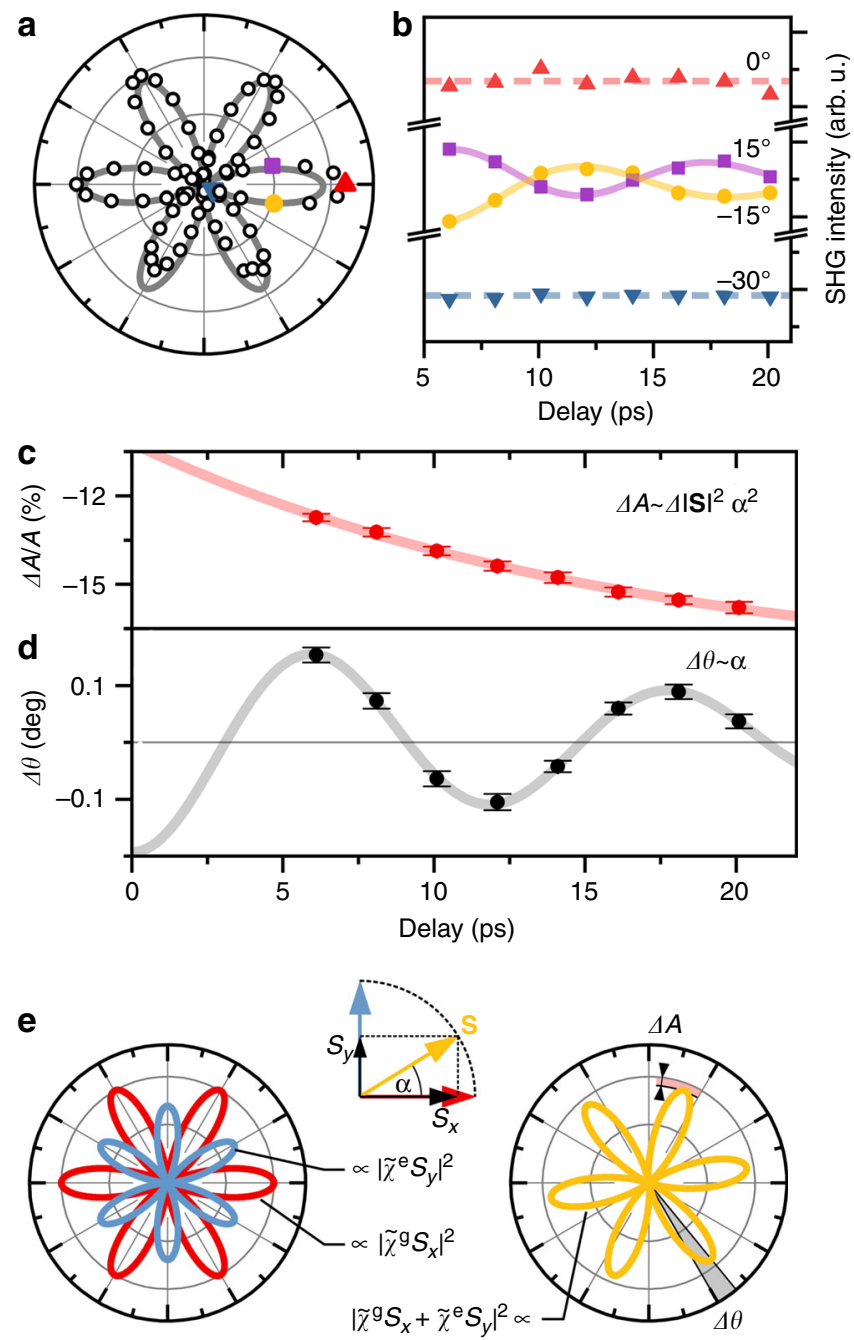

Fig. 3 TR modulation of the SHG anisotropy. a Measured ground state SHG anisotropy. b Time-dependent normalised correlated SHG intensity at different points of the anisotropy (see "Methods"). c Time dependence of the relative amplitude change. $\mathbf{d}$ Time dependent rotation angle of the SHG anisotropy after excitation. Solid lines are fits. The error bars denote standard errors of the means determined from 16 consecutive measurements. e Red and blue curves are calculated SHG anisotropies according to $P G_{3}^{\prime} \mathrm{cm}^{\prime}(\mathbf{S} \| \hat{x})$ and $P G_{3}^{\prime} c^{\prime} m(\mathbf{S} \| \hat{y})$ symmetry, respectively. A spin canted by an angle $\alpha$ leads to a superposition of $S_{x}$ - and $S_{y}$-related SHG contributions. Their interference causes a rotation and amplitude change of the SHG anisotropy (yellow curve)

$\mathcal{X}(t)=\cos \alpha(t) \widetilde{\mathcal{X}}^{g}+\sin \alpha(t) \tilde{\mathcal{X}}^{e}$ with time-independent tensors $\tilde{\mathcal{X}}^{g}$ and $\tilde{\mathcal{X}}^{e}$. Thus, complementary to the Faraday rotation, which is sensitive to the uncompensated $z$ component of the spin precession, the rotation of the SHG anisotropy reflects the transversal dynamics of the compensated spin component in the $x y$ plane. Furthermore, according to the Curie principle ${ }^{40}$, the isotropic effect of a thermal excitation cannot reduce the symmetry of $\mathcal{X}$. Thus, the SHG anisotropy rotation is a pristine measure of the non-thermal spin dynamics. Separating thermal and non-thermal dynamics is therefore possible.

\section{Discussion}

We can use the SHG measurements to quantify the spin deflection angle $\alpha$ according to $3 \Delta \theta \approx \rho \alpha$ (see Supplementary Note 1), where

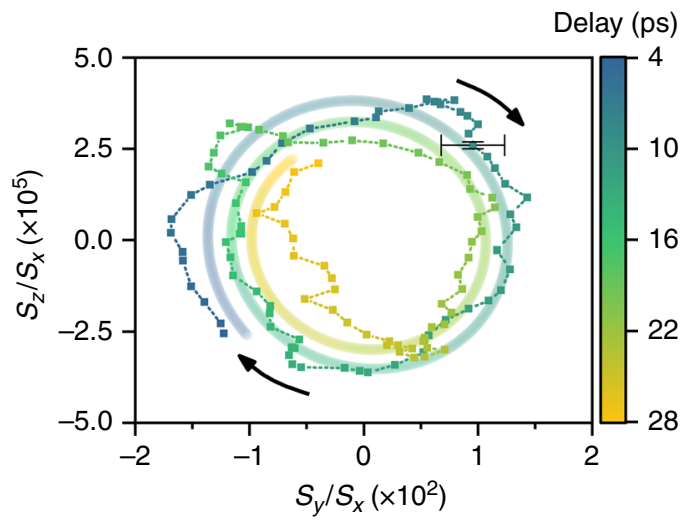

Fig. 4 Magnonic $Z$-mode precession. Time dependent amplitude of the spin motion in the $y z$ plane obtained by combining floating time averages of SHG and Faraday-rotation measurements. The solid line is a fit of a damped elliptical spin precession. For clarity, only two precession periods are shown

$\rho=\left|\widetilde{\mathcal{X}}^{e}\right| /\left|\widetilde{\mathcal{X}}^{g}\right|=0.6$ is the amplitude ratio of the real-valued susceptibilities $\widetilde{\mathcal{X}}_{i j k} 13,41,42$. Note that $\rho$ changes by \pm 0.3 within the probe laser linewidth, which introduces a systematic error of that order. From Fig. 3d, we extrapolate $\Delta \theta(0)=0.198^{\circ} \pm 0.007^{\circ}$ and, hence, $\alpha(0)=1.0^{\circ}$ with a statistical and systematic uncertainty of $0.035^{\circ}$ and $0.5^{\circ}$, respectively. This, in turn, allows us to quantify the effective magnetic field of the IFE as $\alpha(0)=\gamma \mu_{0} H_{\mathrm{IFE}} \tau$, where $1^{\circ}$ corresponds to $\mu_{0} H_{\mathrm{IFE}} \approx 760 \mathrm{mT}$ for $\tau=130 \mathrm{fs}$ (assuming the free electron value $\left.\gamma=176 \mathrm{~s}^{-1} \mathrm{~T}^{-1}\right)$. Note that the optically induced spin canting by non-resonant excitation via the IFE is comparable to resonant excitation via the magnetic field component of a strong $\mathrm{THz}$ pulse ${ }^{43}$.

In addition to the quantification of the basal-plane spin-canting angle, we can use the TR Faraday rotation to estimate the maximum out-of-plane spin-canting angle as $2.4 \mathrm{mdeg}$ (see Supplementary Note 2). Such a highly anisotropic spin precession is a general phenomenon in antiferromagnets and highlights the dominance of the exchange interaction over the weak magnetic in-plane anisotropy ${ }^{8,28}$.

After relating the basal-plane spin-canting angle $\alpha$ and the total sublattice magnetisation $g \mu_{B}|S|$ to the SHG anisotropy orientation and amplitude, respectively, we understand the measurement shown in Fig. 2 as follows: The observed TR SHG response is a superposition of longitudinal and transversal spin dynamics and therefore a combination of scenarios (i) and (ii). The time dependence of $\alpha$, and hence $S_{y}$, is reflected by the periodic SHG modulation. We combine this with the $S_{z}$-sensitive Faraday rotation to obtain the full order-parameter motion. By plotting the transversal spin components in Fig. 4, one can clearly recognise the magnonic damped elliptical motion (solid line).

We thus present a proof-of-concept experiment, where we track the full three-dimensional motion of an AFM order parameter. In our model compound, $\mathrm{YMnO}_{3}, \mathrm{SHG}$ and Faraday rotation are combined to obtain the trajectory of an optically induced coherent spin precession. While the out-of-plane spin canting induces an uncompensated magnetic moment that causes a Faraday rotation, the basal-plane canting and the total sublattice magnetisation are reflected in the SHG measurements. We quantify the optically induced canting as approximately $1^{\circ}$, corresponding to an effective magnetic field of the IFE of $760 \mathrm{mT}$. Key to this analysis is not the symmetry of the magnetic ground state, but the transient symmetry reduction during the coherent excitation of an $A_{2}$ magnon mode. This symmetry reduction directly affects the highly symmetry-sensitive SHG anisotropy and allows us to separate non-thermal spin dynamics from 
thermal electron dynamics. We emphasise though that our approach is not at all limited to harmonic small-amplitude dynamics. Instead, we exploit a fundamental excitation to showcase the insights provided by a TR symmetry analysis, while at the same time establishing a general approach for accessing order-parameter dynamics at sub-picosecond timescales. Tracking the full order-parameter motion instead of just one component is indispensable for understanding the highly complex dynamics occurring during ultrafast switching, spin-reorientation and other non-equilibrium phenomena.

\section{Methods}

Sample. Our $\mathrm{YMnO}_{3}$ sample is a single crystal grown by the floating-zone method. We oriented, cut and polished the sample to obtain a $20 \mu \mathrm{m}$ thin slab with (0001)oriented surfaces for experiments in transmission geometry. The sample was mounted in a cryostat (Janis SVT-400).

Pump-probe experimental setup. The fundamental light source of our setup is a regeneratively amplified Ti:Sapphire laser (Coherent, Legend, $2.5 \mathrm{~W}$ ) producing $130 \mathrm{fs}$ laser pulses at $1 \mathrm{kHz}$ with a central photon energy of $1.55 \mathrm{eV}$. For our measurements, we used the output of two optical parametric amplifiers providing pump and probe pulses, respectively. To ensure excitation by the IFE, the circularly polarised pump pulses were tuned to $0.97 \mathrm{eV}$, where the absorption of $\mathrm{YMnO}_{3}$ is low. A residual absorption coefficient of $\approx 30 \mathrm{~cm}^{-1}$ at the pump photon energy and $\approx 40 \mathrm{~cm}^{-1}$ at the probe photon energy ${ }^{31}$ results in an absorption of $\approx 6 \%$ and $\approx 8 \%$, respectively. The pump fluence was approximately $60 \mathrm{~mJ} \mathrm{~cm}^{-2}$. Linearly polarised probe pulses at $1.22 \mathrm{eV}$ allow for the detection of Faraday rotation and SHG. The pump and probe spots exhibit Gaussian profiles with diameters of approximately 180 and $150 \mu \mathrm{m}$, respectively. In order to observe the SHG modulation in Fig. 2, the linear polarisation was set to $15^{\circ}$ relative to the crystallographic y axis, i.e., to an inclination point of the sixfold antiferromagnetic SHG anisotropy. The transmitted probe pulse passed a dichroic mirror that spatially separated the linear and non-linear optical response. The Faraday rotation was detected using a balanced photodiode, while simultaneously a photomultiplier tube measured the SHG yield in the pumped and unpumped state.

SHG anisotropy measurements. All measurements were performed in nearnormal incidence. The polarisation dependence of the SHG anisotropy was obtained by keeping the sample fixed and rotating the light polarisation of the incoming probe laser pulse with a half-wave plate. The transmission direction of a Glan-Laser prism inserted between the dichroic mirror and the photomultiplier tube (not shown in Fig. 1) was always set perpendicular to the polarisation of the incoming probe light. Each SHG anisotropy consists of 73 individual data points spaced by $5^{\circ}$. The changes of amplitude $\Delta A / A$ and orientation $\Delta \theta$ were extracted from a fit according to

$$
I(\phi)=C+A \cos ^{2} 3(\phi+\theta),
$$

The uncertainties shown in Fig. $3 c$, d denote standard errors of the amplitudes and orientations.

Figure $3 \mathrm{~b}$ is obtained by normalising the SHG anisotropy for each delay time to its maximum, averaging each data point with its symmetry equivalent points, i.e.,

$$
I_{\text {ave }}(\phi)=\frac{1}{6} \sum_{i=0}^{5} I\left(\phi+i * 60^{\circ}\right),
$$

and plotting $I_{\text {ave }}\left(0^{\circ}\right), I_{\text {ave }}\left( \pm 15^{\circ}\right)$ and $I_{\text {ave }}\left(-30^{\circ}\right)$.

\section{Data availability}

The data that support the findings of this study are available from the corresponding author upon reasonable request.

Received: 28 March 2019 Accepted: 6 August 2019

Published online: 05 September 2019

\section{References}

1. Kimel, A. V. et al. Inertia-driven spin switching in antiferromagnets. Nat. Phys. 5, 727-731 (2009).

2. Wadley, P. et al. Electrical switching of an antiferromagnet. Science 351, 587-590 (2016).

3. Jungwirth, T., Marti, X., Wadley, P. \& Wunderlich, J. Antiferromagnetic spintronics. Nat. Nanotechnol. 11, 231-241 (2016).
4. Gomonay, O., Baltz, V., Brataas, A. \& Tserkovnyak, Y. Antiferromagnetic spin textures and dynamics. Nat. Phys. 14, 213-216 (2018).

5. Acremann, Y. et al. Imaging precessional motion of the magnetization vector. Science 290, 492-495 (2000).

6. Tesařová, N. et al. Direct measurement of the three-dimensional magnetization vector trajectory in GaMnAs by a magneto-optical pump-andprobe method. Appl. Phys. Lett. 100, 102403 (2012).

7. Saidl, V. et al. Optical determination of the Néel vector in a CuMnAs thin-film antiferromagnet. Nat. Photon. 11, 91-96 (2017).

8. Tzschaschel, Ch. et al. Ultrafast optical excitation of coherent magnons in antiferromagnetic NiO. Phys. Rev. B 95, 174407 (2017).

9. Zheng, Z. et al. Magneto-optical probe of ultrafast spin dynamics in antiferromagnetic $\mathrm{CoO}$ thin films. Phys. Rev. B 98, 134409 (2018).

10. Sa, D., Valentí, R. \& Gros, C. A generalized Ginzburg-Landau approach to second harmonic generation. Eur. Phys. J. B 14, 301-305 (2000).

11. Fiebig, M., Pavlov, V. V. \& Pisarev, R. V. Second-harmonic generation as a tool for studying electronic and magnetic structures of crystals: review. J. Opt. Soc. Am. B 22, 96-118 (2005).

12. Shen, Y. R. The Principles of Nonlinear Optics. (Wiley-Interscience, Hoboken, 2003).

13. Fiebig, M., Fröhlich, D., Lottermoser, Th. \& Kohn, K. Spin-angle topography of hexagonal manganites by magnetic second-harmonic generation. Appl. Phys. Lett. 77, 4401 (2000).

14. Chauleau, J.-Y., Haltz, E., Carrétéro, C., Fusil, S. \& Viret, M. Multi-stimuli manipulation of antiferromagnetic domains assessed by second-harmonic imaging. Nat. Mater. 16, 803-807 (2017).

15. Huber, L. et al. Coherent acoustic perturbation of second-harmonic generation in NiO. Phys. Rev. B 92, 094304 (2015).

16. Kirilyuk, A., Kimel, A. V. \& Rasing, Th. Ultrafast optical manipulation of magnetic order. Rev. Mod. Phys. 82, 2731-2784 (2010).

17. Satoh, T., Van Aken, B. B., Duong, N. P., Lottermoser, Th. \& Fiebig, M. Ultrafast spin and lattice dynamics in antiferromagnetic $\mathrm{Cr}_{2} \mathrm{O}_{3}$. Phys. Rev. $B$ 75, 155406 (2007).

18. Matsubara, M., Kaneko, Y., He, J.-P., Okamoto, H. \& Tokura, Y. Ultrafast polarization and magnetization response of multiferroic $\mathrm{GaFeO}_{3}$ using timeresolved nonlinear optical techniques. Phys. Rev. B 79, 140411(R) (2009).

19. Sala, V. G. et al. Resonant optical control of the structural distortions that drive ultrafast demagnetization in $\mathrm{Cr}_{2} \mathrm{O}_{3}$. Phys. Rev. B 94, 014430 (2016).

20. Sheu, Y. M., Ogawa, N., Kaneko, Y. \& Tokura, Y. Photocreating supercooled spiral-spin states in a multiferroic manganite. Phys. Rev. B 94, 081107(R) (2016).

21. Satoh, T. et al. Spin oscillations in antiferromagnetic NiO triggered by circularly polarized light. Phys. Rev. Lett. 105, 077402 (2010).

22. Ivanov, B. A. Spin dynamics of antiferromagnets under action of femtosecond laser pulses (Review Article). Low Temp. Phys. 40, 91-105 (2014).

23. Satoh, T., Iida, R., Higuchi, T., Fiebig, M. \& Shimura, T. Writing and reading of an arbitrary optical polarization state in an antiferromagnet. Nat. Photon. 9 , 25-29 (2015).

24. Bossini, D. \& Rasing, Th. Femtosecond optomagnetism in dielectric antiferromagnets. Phys. Scr. 92, 024002 (2017).

25. Satoh, T. et al. Excitation of coupled spin-orbit dynamics in cobalt oxide by femtosecond laser pulses. Nat. Commun. 8, 638 (2017).

26. Němec., P., Fiebig, M., Kampfrath, T. \& Kimel, A. V. Antiferromagnetic optospintronics. Nat. Phys. 14, 229-241 (2018).

27. Lilienblum, M. et al. Ferroelectricity in the multiferroic hexagonal manganites. Nat. Phys. 11, 1070-1073 (2015).

28. Toulouse, C. et al. Lattice and spin excitations in multiferroic h- $\mathrm{YMnO}_{3}$. Phys, Rev. B 89, 094415 (2014).

29. Wang, Y. T., Luo, C. W. \& Kobayashi, T. Understanding multiferroic hexagonal manganites by static and ultrafast optical spectroscopy. $A d v$. Condens. Matter Phys. 2013, 104806 (2013).

30. Kalashnikova, A. M. \& Pisarev, R. V. Electronic structure of hexagonal rareearth manganites $R \mathrm{MnO}_{3}$. J. Exp. Theor. Phys. 78, 143-147 (2003).

31. Babonas, G.-J., Grivel, J.-C., Reza, A. \& Girkantaite, R. Ellipsometric studies of $\mathrm{ErMnO}_{3}$ single crystals. Lith. J. Phys. 47, 309-314 (2007).

32. Degenhardt, C., Fiebig, M., Fröhlich, D., Lottermoser, Th. \& Pisarev, R. V. Nonlinear optical spectroscopy of electronic transitions in hexagonal manganites. Appl. Phys. B 73, 139-144 (2001).

33. Landau, L. D., Pitaevskii, L. P. \& Lifshitz, E. M. Electrodynamics of Continuous Media. (Pergamon, Oxford, 1984).

34. Mikhaylovskiy, R. V. et al. Ultrafast optical modification of exchange interactions in iron oxides. Nat. Commun. 6, 8190 (2015)

35. Sheu, Y. M., Ogawa, N., Tokunaga, Y., Chan, H. C. \& Tokura, Y. Selective probe of coherent polar phonon and quasiferromagnetic resonance modes in multiferroic $\mathrm{GdFeO}_{3}$. Phys. Rev. B 98, 100301(R) (2018).

36. Mankowsky, R., von Hoegen, A., Först, M. \& Cavalleri, A. Ultrafast reversal of the ferroelectric polarization. Phys. Rev. Lett. 118, 197601 (2017). 
37. Melnikov, A. et al. Coherent optical phonons and parametrically coupled magnons induced by femtosecond laser excitation of the $\mathrm{Gd}(0001)$ surface. Phys. Rev. Lett. 91, 227403 (2003).

38. Matsubara, M. et al. Ultrafast optical tuning of ferromagnetism via the carrier density. Nat. Commun. 6, 6724 (2015).

39. Kimel, A. V., Kirilyuk, A., Tsvetkov, A., Pisarev, R. V. \& Rasing, Th. Laserinduced ultrafast spin reorientation in the antiferromagnet $\mathrm{TmFeO}_{3}$. Nature 429, 850-853 (2004).

40. Hargittai, I. \& Vainshtein, B. K. Crystal Symmetries. (Pergamon, Amsterdam, 1989).

41. Iizuka-Sakano, T., Hanamura, E. \& Tanabe, Y. Second-harmonic-generation spectra of the hexagonal manganites $\mathrm{RMnO}_{3}$. J. Phys. 13, 3031-3055 (2001).

42. Lottermoser, Th. Elektrische und Magnetische Ordnung Hexagonaler Manganite. (PhD Thesis, Dortmund, 2002).

43. Baierl, S. et al. Terahertz-driven nonlinear spin response of antiferromagnetic nickel oxide. Phys. Rev. Lett. 117, 197201 (2016).

\section{Acknowledgements}

The authors thank Morgan Trassin and Mads Weber for valuable discussions. T.S. was supported by Japan Society for the Promotion of Science (JSPS) KAKENHI (Nos. JP15H05454, JP19H01828 and JP26103004) and JSPS Core-to-Core Programme (A Advanced Research Networks). C.T. and M.F. acknowledge support from the SNSF project 200021/147080 and by FAST, a division of the SNSF NCCR MUST. M.F. thanks ETH Zurich and CEMS at RIKEN for support of his research sabbatical.

\section{Author contributions}

T.S. conceived the project. C.T. designed, conducted and evaluated the experiments. T.S. and M.F. supervised the project. All authors discussed the results and contributed to the writing of the paper.

\section{Additional information}

Supplementary Information accompanies this paper at https://doi.org/10.1038/s41467 019-11961-9.

Competing interests: The authors declare no competing interests.

Reprints and permission information is available online at http://npg.nature.com/ reprintsandpermissions/

Peer review information: Nature Communications thanks Alireza Qaiumzadeh, and other anonymous reviewer(s) for their contribution to the peer review of this work. Peer reviewer reports are available.

Publisher's note: Springer Nature remains neutral with regard to jurisdictional claims in published maps and institutional affiliations.

(c) (i) Open Access This article is licensed under a Creative Commons Attribution 4.0 International License, which permits use, sharing, adaptation, distribution and reproduction in any medium or format, as long as you give appropriate credit to the original author(s) and the source, provide a link to the Creative Commons license, and indicate if changes were made. The images or other third party material in this article are included in the article's Creative Commons license, unless indicated otherwise in a credit line to the material. If material is not included in the article's Creative Commons license and your intended use is not permitted by statutory regulation or exceeds the permitted use, you will need to obtain permission directly from the copyright holder. To view a copy of this license, visit http://creativecommons.org/ licenses/by/4.0/.

(C) The Author(s) 2019 